\title{
ANALYTICAL EFFECTS OF GRAVITATIONAL WAVES \\ ON THE MOTION OF AN ARTIFICIAL SATELLITE
}

\author{
M. H. YOUSSEF AND M.K. AHMED \\ Astronomy Department, Faculty of Science \\ Cairo, Egypt
}

\begin{abstract}
The motion of an artificial satellite in the Earth's gravitational field is discussed in the post-Newtonian framework including the effect of weak gravitation waves using the perturbation technique of the canonical Lie-transformations. Two successive canonical transformations are used to derive analytical expressions for the short-period, long-period and secular perturbations of orbital elements. The solution is expressed in terms of the Delaunay variables.
\end{abstract}

\section{Introduction}

All methods developed to detect gravitational waves depend more or less on the fact that maximum variation on the separation of two particles, produced by a gravitational wave propagating along the Z-axis, occurs if the particles are located in the XY plane. For a plane wave travelling in the Z-direction the only non-vanishing components are $h_{11}=-h_{22}$ and $h_{12}=h_{21}$ so that the metric describing the wave becomes

$$
d s^{2}=c^{2} d t^{2}-d z^{2}-\left(1-h_{11}\right) d x^{2}-\left(1+h_{11}\right) d y^{2}+2 h_{12} d x d y,
$$

and the separation

$$
d l^{2}=d z^{2}+\left(1-h_{11}\right) d x^{2}+\left(1+h_{11}\right) d y^{2}+2 h_{12} d x d y .
$$

The plane wave can be written as the sum of two components

$$
\begin{aligned}
& h_{11}=h_{t} \cos \left(n_{g} t+\alpha_{1}\right), \\
& h_{12}=h_{x} \cos \left(n_{g} t+\alpha_{2}\right),
\end{aligned}
$$

I. M. Wytrzyszczak, J. H. Lieske and R. A. Feldman (eds.),

Dynamics and Astrometry of Natural and Artificial Celestial Bodies, 431, 1997.

(C) 1997 Kluwer Academic Publishers. Printed in the Netherlands. 
where $n_{g}$ is the frequency of the wave, $\alpha_{1}$ and $\alpha_{2}$ are the phase differences, $h_{t}$ and $h_{x}$ are the amplitudes of the wave in the two orthogonal directions in the transverse plane.

To find acceleration components produced by the wave on a bound system of two bodies (Earth-Satellite) we assume that the characteristic dimension of the system is small compared to the length of the wave, and the velocities of the system are much smaller than the speed of light. The radial accelerations (tidal forces) are described by Riemann tensor via the equation of geodesic deviation (Straumann, 1984)

$$
\frac{d^{2} n^{i}}{d t^{2}}=\frac{1}{2} \frac{\partial^{2} h_{i j}}{\partial t^{2}} n^{j}
$$

where $n$ is the separation vector between neighboring geodesics. In terms of $x, y, z$ coordinates, the equation (5) yields

$$
\begin{aligned}
& \frac{d^{2} x}{d t^{2}}=\frac{1}{2} \frac{\partial^{2} h_{11}}{\partial t^{2}} x+\frac{1}{2} \frac{\partial^{2} h_{12}}{\partial t^{2}} y \\
& \frac{d^{2} y}{d t^{2}}=\frac{1}{2} \frac{\partial^{2} h_{12}}{\partial t^{2}} x-\frac{1}{2} \frac{\partial^{2} h_{11}}{\partial t^{2}} y \\
& \frac{d^{2} z}{d t^{2}}=0 .
\end{aligned}
$$

In the present work, we are using the canonical Lie series methods (Hori, 1966; Deprit, 1969) to define and evaluate the amount of the mixed perturbations produced by the gravitational waves and the oblateness of the Earth. The series, in canonical Delaunay variables, are expanded up to the fourth order of the oblateness parameter $J_{2}$.

\section{The Hamiltonion}

From the components of the acceleration vector at a point with coordinantes $\mathrm{x}, \mathrm{y}, \mathrm{z}$, which is given in (6), we can construct the Hamiltonian of the gravitational waves as follows:

$$
H_{W}=\frac{1}{2} h_{1}\left(y^{2}-x^{2}\right)-h_{2} x y
$$

where $h_{1}=\frac{1}{2} \mu \frac{\partial^{2} h_{11}}{\partial t^{2}}=\beta \cos \left(n_{g} t+\alpha_{1}\right), h_{2}=\frac{1}{2} \mu \frac{\partial^{2} h_{12}}{\partial t^{2}}=\gamma \cos \left(n_{g} t+\alpha_{2}\right)$, $\beta=-\frac{1}{2} \mu h_{t} n_{g}^{2}, \gamma=-\frac{1}{2} \mu h_{x} n_{g}^{2}, \mu$ is the reduced mass of the system, and $\beta$ and $\gamma$ coefficients are assumed to be of order $J_{2}^{3}$.

We can express $H_{W}$ in terms of the Delaunay variables:

$l_{1}=l$ (mean anomaly), $L_{1}=L=\sqrt{\mu a}, l_{2}=g=\omega$ (argument of perigee) $L_{2}=G=\sqrt{\mu a\left(1-e^{2}\right)}, l_{3}=h=\Omega$ (longitude of the node), $L_{3}=H=$ 
$G \cos i$, and expand the functions of the true anomaly in terms of the mean anomaly Hansen's coefficients (Haighara, 1970) so that all powers of the eccentricity can be retained. It is clear, that the Hamiltonian $H_{W}$ depends explicitely on the time through the term $n_{g} t$. To remove the time from the Hamiltonian we set $n_{g} t=l_{4}$, and augment the Delaunay variables by the pair $\left(l_{4}, L_{4}\right)$, where $L_{4}$ is the conjugate of $l_{4}$ which will be found from equation $L_{4}=-\frac{\partial H_{W}}{\partial l_{4}}$, using the unperturbed part of $H_{W}$. Finally, the Hamiltonian becomes

$$
H_{W}=H_{0}+\epsilon H_{1},
$$

where $H_{0}=-\frac{\mu}{2 L^{2}}+n_{g} L_{4}, \epsilon$ is a small parameter assuming that its order is of $O\left(J_{2}^{3}\right)$, and

$$
H_{1}=L_{1}^{4} \sum_{N=-\infty}^{\infty}\left\{\alpha_{N}^{c} \cos \left(N l_{1}+l_{4}\right)+\alpha_{N}^{s} \sin \left(N l_{1}+l_{4}\right)\right\},
$$

where $\alpha_{N}^{c}$ and $\alpha_{N}^{s}$ are coefficients depending on Delaunay variables $\left(l_{2}\right.$, $\left.l_{3}, L_{2}, L_{3}\right)$ and the dimensionless amplitudes of the wave $\left(h_{x} h_{t}^{-1}\right)$. Since the parameters of the wave's potential are much smaller than any of the harmonics of the Earth's potential, we can add the solution obtained from the wave's Hamiltonian, linearly to the results of any artificial satellite theory to find the combined effects of the oblateness of the Earth and of the gravitational waves. Therefore, the Hamiltonian which describes the relative motion of an artificial satellite moving in the Earth's gravitational field and the external wave can be expressed as

$$
H=H_{g}+H_{W},
$$

where $H_{g}$ represents the Hamiltonian of an artificial satellite in the Earth's gravitational field

$$
H_{g}=v P-L,
$$

$\mathrm{L}$ is the Lagrangian of a satellite moving about a central body, in the Einstein form of the post Newtonian gravitational field

$$
L=\frac{1}{2} v^{2}-V_{N}+\frac{1}{c_{l}^{2}}\left\{\frac{v^{4}}{8}-\frac{1}{2} V_{N}^{2}-\frac{3}{2} v^{2} V_{N}\right\}
$$

$v$ is the velocity of the satellite, $c_{l}$ is the speed of light, $\mathrm{P}$ is the canonical momentum, and $V_{N}$ is the gravitational potential.

We truncate the gravitational potential beyond the fourth harmonic and neglect all deviations from axial symmetry. Therefore, we write the Hamiltonian $H_{g}$ in the following form:

$$
H_{g}=H_{0}+\sum_{n=1}^{4} \frac{\epsilon^{n}}{n !} H_{n},
$$


where $\epsilon$ is the small parameter of the expansion which represents the second Zonal harmonic $J_{2}$ of the Earth's potential, and

$$
\begin{aligned}
H_{0} & =-\frac{\mu^{2}}{2 L^{2}} \\
H_{1} & =\frac{A_{1}}{L^{6}}\left(\frac{a}{r}\right)^{3}\left\{\theta_{0}+\theta_{1}+\cos 2(f+g)\right\} \\
H_{2} & =H_{21}+H_{22}+H_{23} \\
H_{21} & =\frac{A_{21}}{L^{8}}\left(\frac{a}{r}\right)^{4}\left\{\theta_{2} \sin (f+g)+\theta_{3} \sin 3(f+g)\right\} \\
H_{22} & =\frac{A_{22}}{L^{10}}\left(\frac{a}{r}\right)^{5}\left\{\theta_{4}+\theta_{5} \cos 2(f+g)+\theta_{6} \cos 4(f+g)\right\} \\
H_{23} & =\frac{A_{23}}{L^{4}}\left\{3\left(\frac{a}{r}\right)^{2}-\left(\frac{a}{r}\right)+\frac{1}{8}\right\} \\
H_{3} & =\frac{A_{3}}{L^{8}}\left\{\frac{1}{2}\left(\frac{a}{r}\right)^{4}-\frac{3}{8}\left(\frac{a}{r}\right)^{3}\right\}\left\{\theta_{0}+\theta_{1}+\cos 2(f+g)\right\} \\
H_{4} & =H_{41}+H_{42}+H_{43} \\
H_{41} & =\frac{A_{41}}{L^{10}}\left\{\frac{1}{4}\left(\frac{a}{r}\right)^{5}-\frac{3}{16}\left(\frac{a}{r}\right)^{4}\right\}\left\{\theta_{2} \sin (f+g)+\theta_{3} \sin 3(f+g)\right\} \\
H_{42} & =\frac{A_{42}}{L^{12}}\left\{\frac{1}{32}\left(\frac{a}{r}\right)^{6}-\frac{3}{128}\left(\frac{a}{r}\right)^{5}\right\}\left\{\theta_{4}+\theta_{5} \cos 2(f+g)+\theta_{6} \cos 4(f+g)\right\} \\
H_{43} & =\frac{A_{43}}{L^{12}}\left(\frac{a}{r}\right)^{6}\left\{\theta_{7}+\theta_{8} \cos 2(f+g)+\theta_{9} \cos 4(f+g)\right\} .
\end{aligned}
$$

In which $(L, G, H, l, g, h)$ are usual Delaunay variables, $\mu$ is the product of the constant of gravitation and the central body's mass, $r$ is the radial distance, $f$ the true anomaly of the satellite, $a$ the semi-major axis of the instantaneous elliptic orbit. For the different orders of $J_{2}$, the coefficients A are:

$$
\begin{aligned}
& A_{1}=\frac{\mu^{4} R^{4}}{4}, \quad A_{21}=J_{3} \frac{\mu^{5} R^{3}}{4 J_{2}^{2}}, \quad A_{22}=J_{4} \frac{\mu^{6} R^{4}}{32 J_{2}^{2}}, \quad A_{23}=-2 ! \frac{\mu^{6} R^{2}}{c_{l}^{2} J_{2}^{2}}, \\
& A_{3}=3 ! \frac{\mu^{6} R^{2}}{c_{l}^{2} J_{2}^{2}}, \quad A_{41}=4 ! J_{3} \frac{\mu^{7} R^{3}}{c_{l}^{2} J_{2}^{4}}, \quad A_{42}=4 ! J_{4} \frac{\mu^{8} R^{4}}{c_{l}^{2} J_{2}^{4}}, \quad A_{43}=3 \frac{\mu^{8} R^{4}}{4 c_{l}^{2} J_{2}^{2}},
\end{aligned}
$$

with $\mathrm{R}$ being the mean equatorial radius of the central body.

Also, in the Hamiltonian $H_{g}$, we find that

$$
\begin{array}{lll}
\theta_{0}=1-3 c^{2}, & \theta_{1}=-3 s^{2}, & \theta_{2}=15 s^{3}-12 s^{2}, \\
\theta_{3}=-5 s^{3}, & \theta_{4}=9-90 c^{2}-105 c^{4}, & \theta_{5}=-20+160 c^{2}-140 c^{4}, \\
\theta_{6}=35 s^{4}, & \theta_{7}=\frac{27}{2} c^{4}-15 c^{2}+\frac{11}{2}, & \theta_{8}=-6+24 c^{2}-18 c^{4}, \\
\theta_{9}=\frac{9}{2} s^{4}, & &
\end{array}
$$

where $c=\cos i, s=\sin i$. 
The second-order term in the Hamiltonian $H_{g}$ which is represented by equation (15) is consisting of the non-relativistic terms $H_{21}$ and $H_{22}$ (given by $J_{3}$ and $J_{4}$ ) and the relativistic term $H_{23}$ (which contains the factor $c_{l}^{-2}$ ). The order of magnitude of each term is given by $A_{21}, A_{22}$ and $A_{23}$ coefficients.

The third-order term $-H_{3}$ in $H_{g}$, given by $J_{2}^{2} c_{l}^{-2}$, and representing the mixed perturbation due to Newtonian quadrupole field and Schwarzschild acceleration, is of order $J_{2}^{3}$. If the terms containing the factor $c_{l}^{-2}$ in $H_{g}$ are omitted, it remains what is usually called the main problem in artificial satellite theory. This is the problem which was solved by Brouwer (1959).

To solve the canonical equations of motion for the Delaunay variables by means of the canonical Lie transformations, we construct two canonical transformations to eliminate the angle variables from the Hamiltonian (10). Firstly, we eliminate the short-period terms (i.e. those depending on $l$ ). The remaining long-period problem is then governed by a Hamiltonian depending only on $L^{\prime}, H^{\prime}, G^{\prime}$ and $g$, where the primes indicate the transformed variables using a second canonical transformation to remove the long-period terms (i.e. those depending on $g^{\prime}$ ). Finally, the remaining secular problem, being independent of all angle variables, can be very simply solved. All calculations of the short-period, long-period and secular terms, up to order four in $J_{2}$, were done on the computer VAX 651 0/VMS V5.5-2 of the Egyptian Universities Network main center at the supreme council of Universities, using the algebraic manipulation language Reduce V3.1 (Youssef, 1994).

\section{Discussion and Conculsion}

Soffel et al. (1988) and Heimberger et al. (1990), have investigated the relativistic effects on the motion of a satellite moving arround an oblate body. Soffel et al. used Gauss' form of the planetary equations, and determined the secular, the short-periodic and the long-periodic relativistic perturbations using the Keplerian elements to the first order in $J_{2}$. Heimberger et al. (1990) have treated the same problem using the canonical Lie series methods. In addition, an important second-order mixed perturbation $J_{2} c_{l}^{-2}$ due to Newtonian quadrupole field and Schwarzschild acceleration, was included in their analysis.

In the present work, we are treating the same problem using the canonical Lie series methods. We are calculating, in addition to Heimberger's work, the third-order, mixed perturbation $J_{2}^{2} c_{l}^{-2}$, and fourth-order mixed $J_{2}^{4} c_{l}^{-4}$ pertubation. This was done to allow the coupling of both effects of gravitational waves and the Earth's oblatness.

Since we have eliminated the short-period terms and the long-period terms from $H_{W}$ up to order four in $J_{2}$, we have found that: 
1. the zero order is given by:

$$
H_{0}=-\frac{\mu}{2 L_{1}^{2}}+n_{g} L_{4}^{2}
$$

2. the first order $\left(H_{1}\right)$ is given by the first order solution of $H_{g}$ coming from the effect of Earth's oblateness.

3 . the second order $\left(H_{2}\right)$ is given by the second order solution of $H_{g}$ coming from the effects of Earth's gravity.

4. the third order solution comes from $H_{W}$, and

5. the fourth order solution represents the coupling between the effect of oblateness and the effect of the wave.

The long-period terms will be never eliminated from the Hamiltonian $H_{W}$. The reason for this is that $l_{4}$ appears only in the terms arising from the wave effects. They can be removed when the following conditions are satisfied:

- when the effect of the wave is not coupled to any other effect. It is clear that this decoupling will occur only in the order 6 in $J_{2}$.

- due to commensurability between the wave frequency and the mean motion of the satellite, when $-N l_{1}=l_{4}$.

\section{References}

Brouwer, D. : 1959, "Solution of the problem of artificial satellite theory without Drag", Astron. J. 64, 378.

Deprit, A.: 1969, "Canonical transformations depending on a small parameter", Celest. Mech. 1, 12.

Heimberger J., Soffel, M., and Ruder, H.: 1990, "Relativistic effects in the motion of artificial satellites: The oblateness of the central body II", Celest. Mech. 47, 205.

Hori, G.: 1966, "Theory of general perturbations with unspecified canonical variables", Publ. Astron. Soc. Jpn. 18, 287.

Hagihara, Y.: 1970, Dynamical Principles and Transformation Theory, Maple press, USA.

Soffel, M., Wirrer, R., Schastok, J., Ruder, H. and Schneider, M.: 1988, "Relativistic effects in the motion of artificial satellites: The oblateness of the central body. I", Celest. Mech. 42, 81.

Straumann, N.: 1984 General Relativity and Relativistic Astrophysics, Springer, Berlin.

Youssef, M.: 1994, The effect of Gravitational Waves on the Motion of Artificial Satellites, Ph.D. thesis, Astronomy Dept., Cairo University, Egypt. 